University of Nebraska - Lincoln

DigitalCommons@University of Nebraska - Lincoln

2009

\title{
The development of jaw motion for mastication
}

Erin M. Wilson

University of Wisconsin-Madison

Jordan R. Green

University of Nebraska-Lincoln, jgreen4@unl.edu

Follow this and additional works at: https://digitalcommons.unl.edu/specedfacpub

Part of the Special Education and Teaching Commons

Wilson, Erin M. and Green, Jordan R., "The development of jaw motion for mastication" (2009). Special Education and Communication Disorders Faculty Publications. 3.

https://digitalcommons.unl.edu/specedfacpub/3

This Article is brought to you for free and open access by the Department of Special Education and Communication Disorders at DigitalCommons@University of Nebraska - Lincoln. It has been accepted for inclusion in Special Education and Communication Disorders Faculty Publications by an authorized administrator of DigitalCommons@University of Nebraska - Lincoln. 
Published in Early Human Development 85:5 (May 2009), pp. 303-311; doi: 10.1016/j.earlhumdev.2008.12.003

Copyright @ 2008 Elsevier B.V. Used by permission. http://www.elsevier.com/locate/earlhumdev

Submitted June 24, 2008; revised December 9, 2008; accepted December 10, 2008; published online January $29,2009$.

\title{
The development of jaw motion for mastication
}

\author{
Erin M. Wilson \\ Waisman Center, University of Wisconsin-Madison
}

Jordan R. Green

Department of Communication Disorders and Special Education, University of Nebraska-Lincoln

Corresponding author - E. M. Wilson, University of Wisconsin-Madison, 446 Waisman Center, 1500 Highland Avenue, Madison WI 53705, USA; tel 608 265-5870, email emhillman@wisc.edu

\begin{abstract}
The developmental course of early chewing has rarely been studied, although such knowledge is essential for understanding childhood feeding and swallowing disorders. The goal of this investigation was to quantitatively describe age- and consistency-related changes in jaw kinematics during early chewing development. An optical-motion tracking system was used to record jaw movements during chewing in 3-dimensions in 11 typically-developing participants longitudinally from 9-30 months of age. Age related changes in jaw movement were described for both puree and regular consistencies. The findings demonstrated that the development of rotary jaw motion, jaw motion speed, and management of consistency upgrades are protracted across the first two years of life. Young children did not differentiate their jaw closing speeds for puree and regular consistencies until 18-24 months of age, at which age the speed of movement was significantly slower for the puree than for the regular consistency. Horizontal jaw closing speed decreased significantly with age for the puree consistency, but not for the regular consistency. The emergence of a rotary chew pattern was not observed at the ages studied.
\end{abstract}

Keywords: deglutition, chewing, mastication, development, kinematics, jaw, consistency

\section{Introduction}

Jaw motion for chewing undergoes considerable reorganization in early childhood secondary to a variety of factors including anatomic and physiologic development as well as the progressive introduction of solid food consistencies. Although knowledge about typical chewing development is essential for understanding childhood feeding and swallowing disorders, little is known about the developmental course of early chewing. Studies of chewing motor development in children have rarely been conducted because recording jaw muscle activity and tracking jaw motion in very young children is difficult. The small number of existing investigations [1-4], suggest that, similar to mature chewing [5, 6], early chewing is characterized by regular patterns of reciprocal activation between antagonistic muscle pairs [2]. Green and colleagues posited that "the general coordinative organization is well established by 12 months of age but continues to be refined during early development" [2, p. 2711].

In the absence of detailed physiologically based studies, knowledge of chewing development has been derived primarily from video recordings or live observations of chin movements (detailed below). Common to most of these descriptive studies is the assertion that the transition toward mature chewing is marked by a shift from a vertical to a rotary pattern of jaw movement. This change in the chewing pattern has been described to occur primarily during the first 24 months of life [7], followed by a period of refinement.
The extant literature on chewing development suggests that the earliest (i.e., 4-6 months of age) jaw motions for chewing are constrained to the inferior-superior dimension of the maxillaryocclusal plane (see Figure 1, Panel A). Specifically, Bosma reported that "initial chewing gestures are of simple mandibular elevation" [8, p. 271], and Sheppard and Mysak suggested that early chewing "consisted of cycles of mandible elevation and depression in combination with various lip and tongue movements" [9, p. 839-840]. Pridham also reported that "at about 6 months of age, infants begin a munching type of oral-motor activity, using up-and-down movements of the jaw" [10, p. S175], a finding which was also confirmed by Arvedson [11] and Arvedson and Lefton-Greif [12].

The next stage in the development of chewing is purportedly marked by the emergence of lateral jaw motion. According to Arvedson, Rogers, and Brodsky, "the vertical movements become associated with alternating lateral motions" ([13], p. 46) of the jaw. The emergence of lateral motion has been confirmed by a number of investigators [8-11]. The final stage in the sequence of chewing development is characterized by the emergence of a rotary motion of the jaw (see Figure 1, Panel B). This characteristic rotary motion has been referenced as the hallmark of mature mastication and is reportedly seen as early as 18 months of age [12] and established by 24-30 months of age [14].

These developmental changes in jaw motion for chewing do not only represent refinement in masticatory control, but also the child's response to the texture and viscoelastic properties of new foods. Although the effect of bolus consistencies on mastica- 

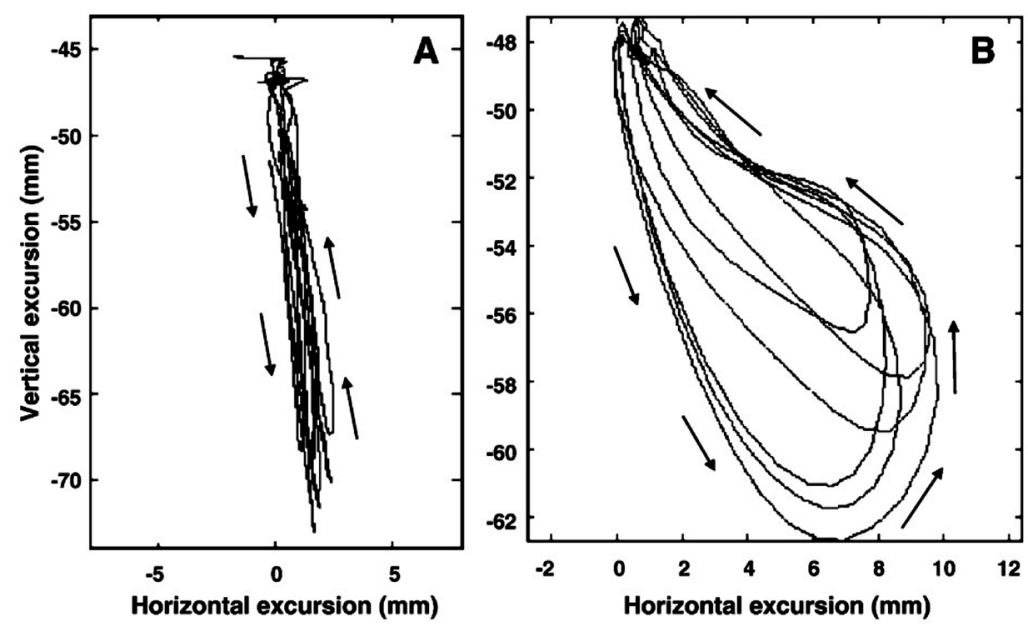

Figure 1. A 2-dimensional representation of the proposed motion of the jaw during two distinct stages of chewing development. The tracings illustrate the predicted motion paths of the jaw during early chewing (Panel A) and mature chewing (Panel B). The x-dimension represents horizontal (side-to-side) motion and the y-dimension represents vertical (up-and-down) motion of the jaw during chewing. Panel A: Formulated kinematic example of jaw motion for early chewing. Note the strict vertical component of the jaw tracing; there is virtually no horizontal contribution. Panel B: Actual kinematic example jaw motion for mature chewing. Note the consistent rotary motion which is characteristic of mature chewing.

tory kinematics and force have been well-documented in adults [15-23], little is known about how immature mandibular control is adapted to accommodate the progressive introduction of new food consistencies. To our knowledge, changes in the spatial aspects of mandibular motion that underlie young children's increased ability to manage different consistency with age have not been described. The few existing studies suggest that consistency contributes significantly to masticatory timing in children; for example, the duration of a chewing sequence is longer for a solid bolus than for a puree bolus [24-27].

The goals of this project are to longitudinally describe age- and consistency-related changes in jaw motion for chewing in 11 typically-developing children from 9 to 30 months of age. Recently developed optical-motion tracking technology was used to record jaw movements during chewing in 3-dimensions [28]. The following experimental questions are posed: (1) Does movement speed for jaw motion increase with age?, (2) Does the relative contribution of the horizontal component of jaw motion during chewing increase with age?, and (3) Are there consistency-related effects on early chewing kinematics? If so, do these effects change with age? If a similar developmental course is seen across the consistencies, the findings can be attributed to changes in motor control and oromandibular biomechanics such as anatomic growth and the emergence of teeth. The results of this kinematic investigation will not only advance our knowledge of the development of motor control for mastication, but in the future, may provide an empirical basis for gauging the severity of feeding disorders.

\section{Methods}

\subsection{Participants}

Eleven children (8 female; 3 male) were studied longitudinally at 9-, 12-, 18-, 24-, and 30-months of age during a 30-45 minute experimental session. The age groups were chosen to represent the stages in chewing development that have been identified in prior investigations. The children in this study were part of a larger, longitudinal investigation on oromotor development from 330 months of age.

\subsection{Age-appropriate development}

All infants were born at term with no neurological, vision, hearing, or physical impairments. At each age level, each infant was seen twice, once for developmental testing and once for orofacial motion capture. During the developmental testing session, each participant was administered the Battelle Developmental Inventory, 2nd edition [29] by a certified speech-language pathologist. This standardized test includes subtests in receptive and expressive communication skills, gross and fine motor skills, and cognitive skills. All children had to score within normal limits at every testing interval for their data to be included in the final data corpus.

\subsection{3-Dimensional motion capture system}

Parents/caregivers were asked to feed their children as they normally would while the motion capture system (Motion Analysis with Eagle Cameras) [30] recorded the movements of the jaw markers in 3-dimensional (3D) space at 120 frames per second. The system consisted of eight infrared cameras and a workstation that computed the 3D positions of the movement markers based on images provided by the eight two-dimensional views. The resultant 3D kinematic signals were digitally low-pass filtered $\left(f_{\mathrm{lp}}=10 \mathrm{~Hz}\right)$ using a zero-phase shift forward and reverse digital filter (Butterworth, 8 pole).

\subsection{Markers}

In order to track the motion of the jaw, small reflective markers, approximately $2 \mathrm{~mm}$ in diameter, were adhered to specific facial landmarks using hypoallergenic tape (see Figure 2). The reflective markers were illuminated with an infrared light source attached to each camera. The marker set consisted of a reference head marker array, used to create an anatomically-based coordinate system (described below), and three jaw markers. The three jaw markers were placed on the chin; one was placed at the gnathion (JC) and two were placed approximately $2 \mathrm{~cm}$ to the right and left of the gnathion marker (JR and JL respectively). Only the JR or the JL marker was used for analysis. These markers were used because, in comparison to markers located near the chin's midline (i.e., JC), skinmotion artifacts are reduced for markers near the lateral regions of the chin [31]. During speech, the average expected error in tracking for markers placed laterally on the chin is approximately $1.08 \mathrm{~mm}$ for the anterior/posterior dimension and $1.89 \mathrm{~mm}$ for the vertical dimension. This amount of error is acceptable, particularly for studies of jaw motion in young children, where there are currently no other existing methods available. 

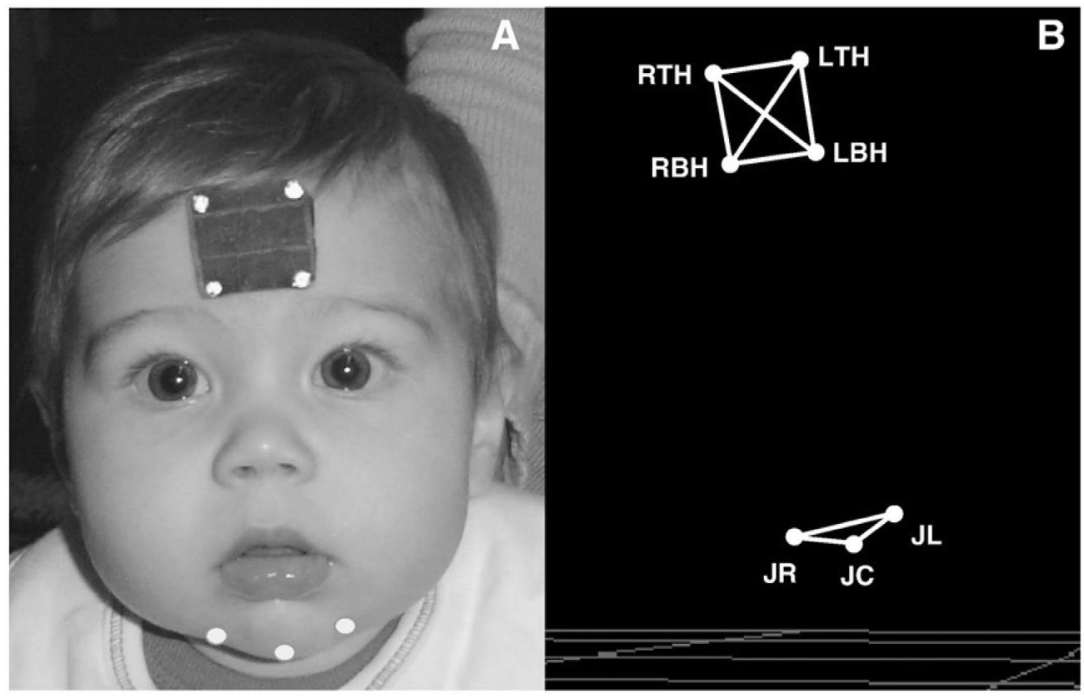

Figure 2. Panel A: Marker set orientation. Panel B: 3-dimensional reconstruction of marker set.

\subsection{Anatomically-based coordinate system}

The predefined anatomically-based coordinate system for this investigation was based on planes oriented about the participant's forehead, and was defined by the rigid head marker array (see Figure 3). The $Y$ axis, or vertical axis, was defined as the line formed by RTH-RBH; the $\mathrm{Z}$ axis was orthogonal to the $\mathrm{Y}$ axis, and the $X$ (or horizontal axis) was formed by the line from RBH to LBH, making the $\mathrm{X}$ axis perpendicular to both the $\mathrm{Y}$ and the $\mathrm{Z}$ axes. All of the analyses were based on this standardized anatomicallybased coordinate system. The head markers were also used to remove translational and rotational components of the head motion from the chin movement data.

\subsection{Bolus consistency}

Food was provided by either the investigator or the parent/caregiver and strict criteria were used to guide the categorization of consistencies based on the National Dysphagia Diet [32]. The two consistencies trialed were puree (e.g., baby cereal, applesauce) and

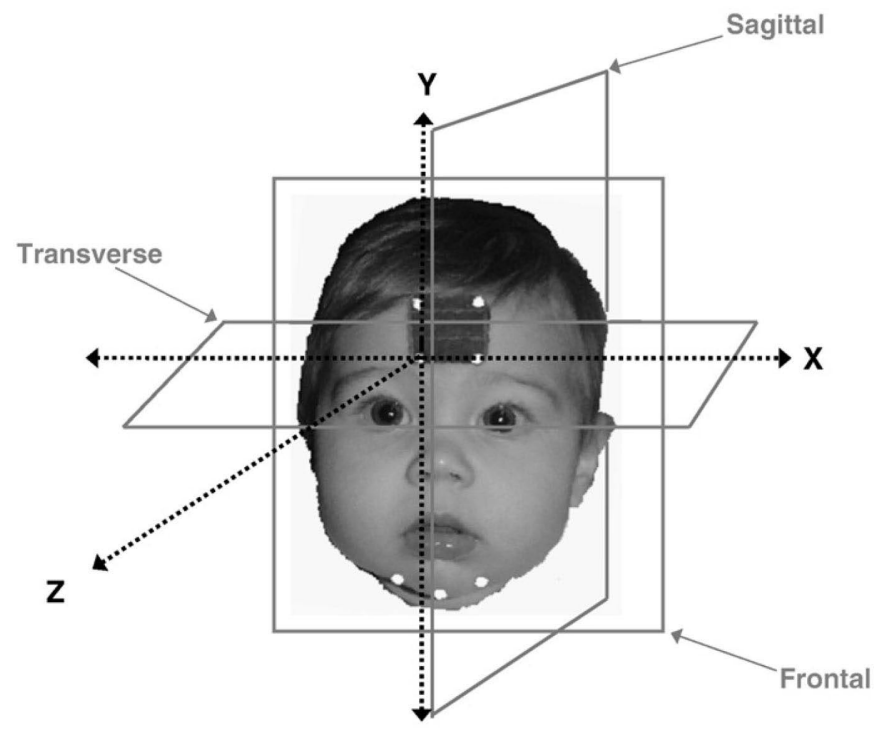

Figure 3. Illustration of the anatomically-based coordinate system used in this investigation. The analyses were based on this standardized anatomically-based coordinate system. regular (e.g., dry cereal). The changes in jaw kinematics in this investigation provide information regarding the expected natural progression of jaw performance for chewing, including those changes that were due to consistency upgrades with age. (Please note the term "consistency upgrades" will be used throughout the manuscript to denote the transition from puree consistency to regular consistency). A standard number of 5 trials of each consistency were attempted with each child; however, due to noncompliance, not all consistencies were accepted by each participant at all of the ages studied (see Table 1).

\subsection{Bolus size}

Bolus size ( $\sim 1 / 2$ tsp for puree; 1 Cheerio for regular bolus) was standardized across subjects and consistencies. The parents or investigators administered each bolus after receiving specific instructions about bolus size requirements. The investigators monitored the parents during feeding to ensure they were administering the appropriate bolus size. Bolus size was also monitored in the review of the digital video recordings during the data parsing process (described below) and trials were excluded accordingly. As a result, 16 chewing trials containing an obviously larger bolus (e.g., > 1/2 tsp) were excluded from the final data set.

\subsection{Signal editing}

The continuous streams of movement data were initially parsed into separate chewing sequences based on digital video record-

Table 1. Distribution of chewing sequences across age groups, subjects, and consistency categories.

\begin{tabular}{llllll}
\hline & Puree & $(n)$ & Regular & $(n)$ & $\%$ \\
\hline 9-month olds & 9 & 2 & 31 & 9 & 22 \\
12-month olds & 12 & 5 & 23 & 9 & 40 \\
18-month olds & 27 & 7 & 24 & 7 & 40 \\
24-month olds & 36 & 9 & 38 & 11 & 82 \\
30-month olds & 40 & 9 & 47 & 11 & 82
\end{tabular}

The numbers in the Puree and Regular columns represent the total number of trials observed for each consistency at the corresponding age range. The $(n)$ columns represent the number of participants observed trialing the consistencies. The \% column represents the percentage of time each participant accepted both consistencies at each time point. 

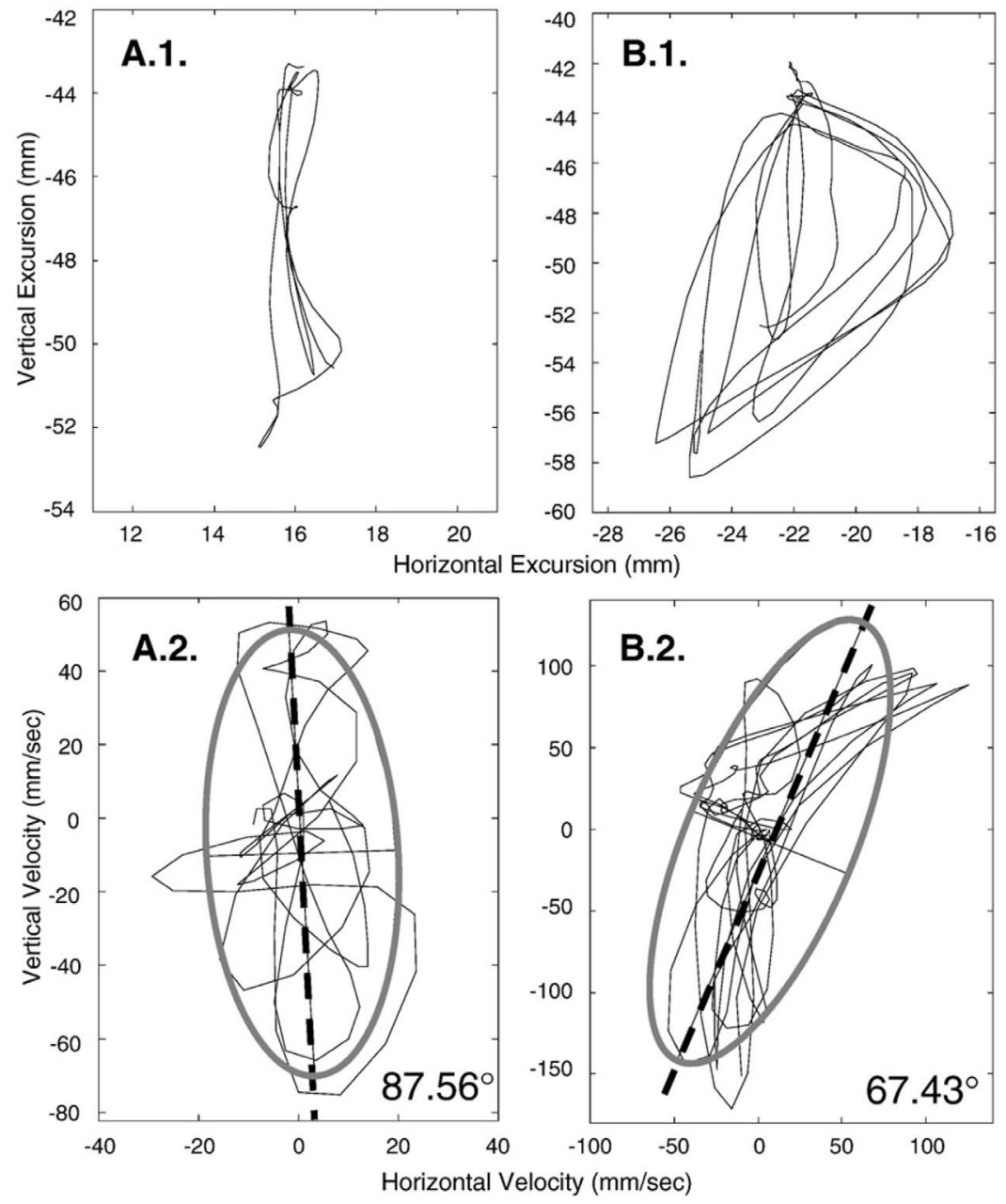

Figure 4. Panel A.1. Exemplar of hypothetical early jaw motion for chewing in the frontal plane. Note the obvious vertical dominance. Panel A.2. Velocity tracing of the jaw motion in A.1. fit with a $2 \mathrm{SD}$ ellipse. The angle of the first principal component (dashed line) is $87.56^{\circ}$ reflecting the obvious vertical dominance of the motion path. Panel B.1. Exemplar of mature (i.e., adult) jaw motion for chewing in the frontal plane. Note the obvious elliptical pattern suggesting a comparatively greater horizontal component. Panel B.2. Velocity tracing of jaw motion in B.1. fit with a 2 SD ellipse. The angle of the first principal component (dashed line) is $67.43^{\circ}$ reflecting the increase in horizontal motion with the elliptical chewing pattern. Recall that a numeric value approximating $90^{\circ}$ represents a predominately vertical chewing sequence; numeric values that deviate from $90^{\circ}$ reflect the emergence of a horizontal component of a chewing sequence.
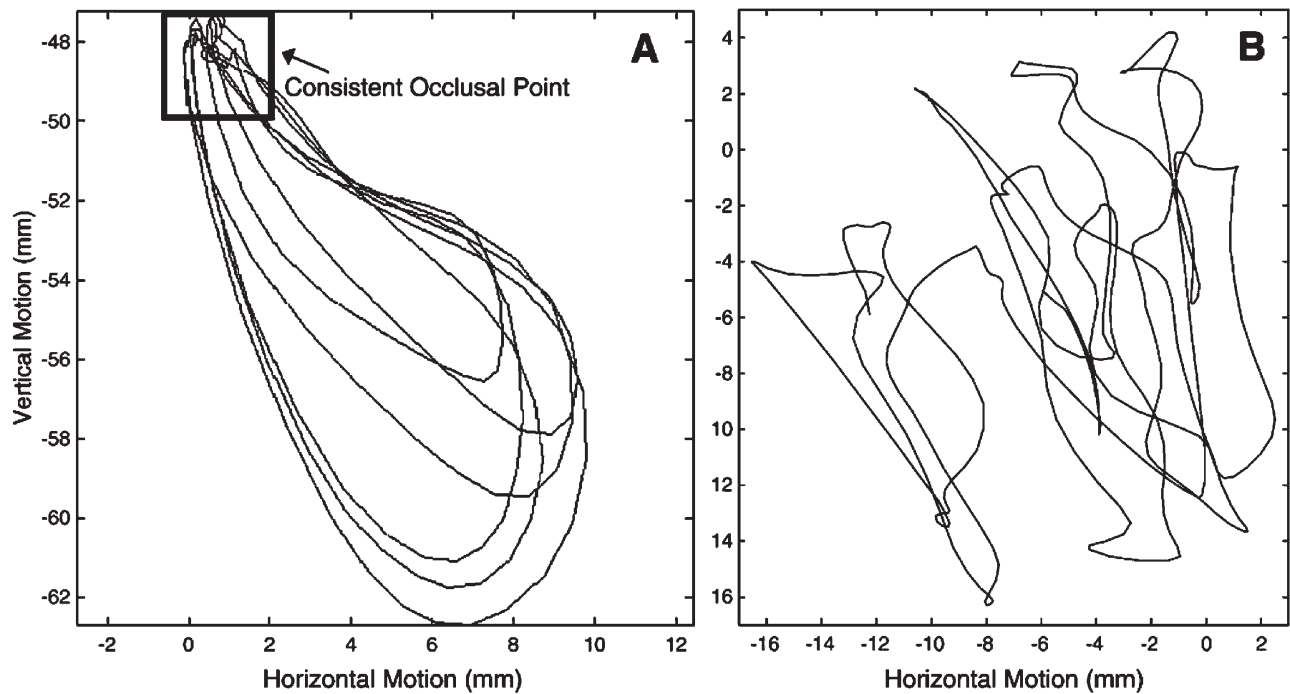

Figure 5. Panel A: Illustration of the horizontal excursion analysis using a mature chewing sequence. Note the consistent occlusal point. Panel B: Illustration of the horizontal excursion analysis using a 12 month old chewing sequence. Note the lack of a consistent occlusal point. 


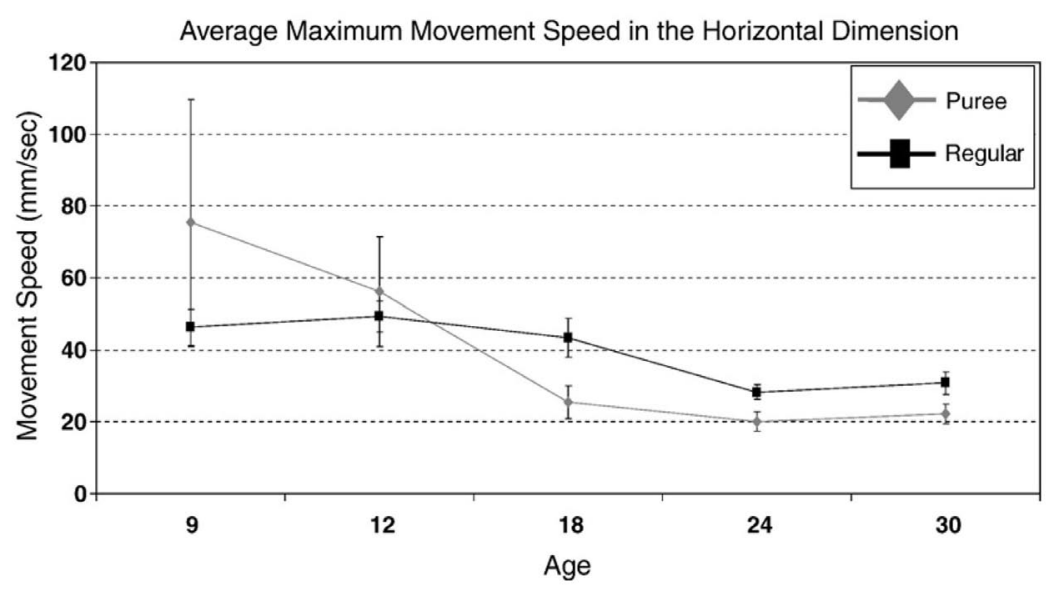

Figure 6. Figure of the average maximum horizontal jaw-closing speed from 9-30 months of age across both consistency categories. The error bars represent average standard error across participants.

ings that were synchronized with the kinematic data. Each chewing sequence was parsed in two stages. The first parsing was intended to identify the entire chewing sequence from the time of maximal jaw closure after the spoon had been removed from the mouth to the time immediately after the swallow. In order to be included as a chewing sequence, each regular consistency trial needed to contain at least three chewing cycles; however, puree trials did not have the same requirement since children often did not require three cycles to breakdown a puree bolus. In the second stage of parsing, each chewing recording was further trimmed by parsing the mid $90 \%$ of the sequence for puree consistency trials and the mid $80 \%$ for regular consistency trials. The resulting mid section (mid $80-90 \%$ ) of each sequence was analyzed. The additional parsing (i.e., mid $80-90 \%$ ) was done to eliminate spurious movements related to positioning of the bolus following placement and/or clearing of the gums/teeth prior to the swallow. If a spurious movement was visually detected anywhere in the remaining mid $80-90 \%$ of a sequence, the file was further parsed to exclude the movement. This additional editing was required for 12 of the 287 files $(4.18 \%)$.

\subsection{Missing data}

Positional data from the jaw marker was occasionally missing when it was covered with food or when it was not within the view of at least two of the cameras. Data was only included in the final data corpus if at least $75 \%$ of the chewing sequence was present; only 1 file was excluded because it contained less than $75 \%$ of the sequence.

\subsection{Data analysis}

\subsubsection{Maximum jaw-closing speed}

The maximum horizontal and vertical speed during jaw closure was calculated for each chewing sequence to determine how jaw movement speed changes as a function of age and consistency. The literature on early motor development suggests that, with some exceptions [33], the speed of limb and oral movements increase with age [34-36]. Therefore, we anticipated movement speed to increase with age, particularly in the horizontal dimension with the emergence of the rotary pattern. We also anticipated an increase in movement speed as a function of consistency upgrades based on findings from Arizumi's investigation of adult chewing [19].

2.10.2. Relative contribution of horizontal and vertical jaw motion For each chewing sequence, the horizontal velocity was plotted as a function of vertical velocity (see Figure 4) to quantify the relative change of each dimension to the jaw motion path as a function of age- and consistency. ${ }^{1}$ To determine the predominant orientation of motion, each velocity plot was fit with a 2-standard deviation ellipse. The angle of the first principal component of that ellipse was then calculated, providing a numeric value that captured the contribution of horizontal and vertical motion to each chewing sequence. To simplify the interpretation of the results (because the orientation of the angle can range from $0-180^{\circ}$ ), the principal component angles were constrained to fall between $0-90^{\circ}$ using the following rule: if $x^{\circ}>90^{\circ}$, then $180^{\circ}-x^{\circ}$. In this analysis, angles approximating $90^{\circ}$ indicated the predominance of a vertical component and angles approximating $45^{\circ}$ indicated an equal contribution from vertical and horizontal components of movement to the jaw motion path. Empirical support for the emergence of a horizontal component in jaw motion during chewing would be provided by the observation that the angle decreases (relative to $90^{\circ}$ ) with age.

Lundeen and Gibbs [18] reported that an increase in lateral (i.e., horizontal) motion corresponds with an increase in bolus consistency. That is, horizontal motion reportedly increases as a function of bolus hardness. Therefore, along with an increase in speed, we also anticipated an increase in the horizontal component with consistency upgrades.

\subsection{Statistical analysis}

Hierarchical linear modeling (HLM) was used to determine (1) the influence of age and consistency on mandibular movement speed and (2) the relative contribution of horizontal and vertical motion of the mandible for each chewing sequence. Separate intercept and slope trajectory parameters were specified for puree and regular consistencies such that each child was assumed to possess a distinct trajectory for each consistency type. The model thus permits a description of the average trajectory (across children) for each consistency type, as well as quantification of the variability between children both within and between consistency types.

\section{Results}

A total of 287 chewing sequences were evaluated (see Table 1). Descriptive statistics for the analyses are displayed in Figures 6-8.

1 Vertical and horizontal displacement was not used because the position of the jaw tends to shift across chewing cycles in young children (see Figure 5). This float has the potential to inflate range of motion estimates. Velocity signals are less sensitive to such positional changes of the jaw. Consequently, plotting the horizontal and vertical velocity traces of a chewing sequence should reveal the relative contribution of motion in each dimension as it is well documented that velocity scales with displacement [37]. 


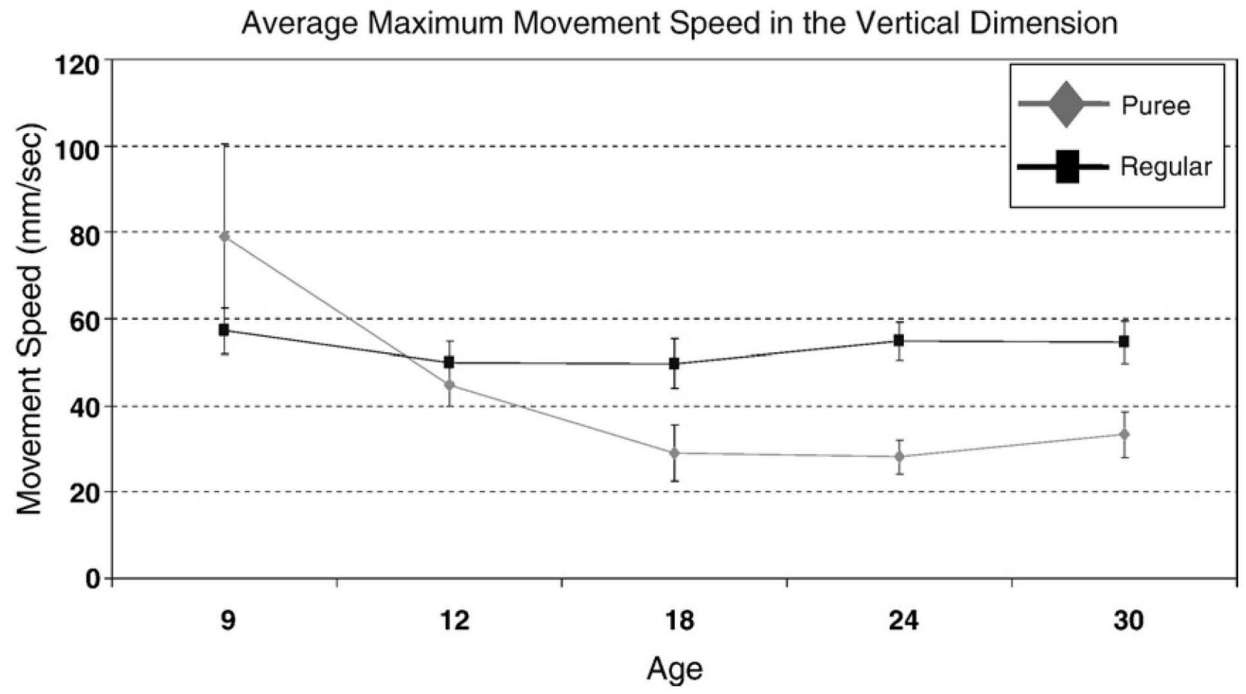

Figure 7. Figure of the average maximum vertical jaw-closing speed from 9-30 months of age across both consistency categories. The error bars represent average standard error across participants.

\subsection{Age effects}

3.1.1. Maximum horizontal jaw-closing speed As depicted in Figure 6, the maximum horizontal closing speed decreased significantly with age for the puree consistency category $(\gamma=-7.20 ; t=-3.61, d f=10, p<0.01)$. A significant quadratic trend $(\gamma=0.22 ; t=3.12, d f=10, \mathrm{p}<0.05)$ was also detected for the puree consistency category, which again indicated a decrease in jaw closing speed with age, but a slowing of the decrease as age increases. No significant trend (i.e., linear or quadratic) was detected for the regular consistency category.

\subsection{Maximum vertical jaw-closing speed}

A pattern similar to that observed for maximum horizontal closing speed was also observed for maximum vertical closing speed for the puree consistency category; however, both the linear and quadratic trends fell below significance. No significant age trend (i.e., linear or quadratic) for maximum closing speed was detected for the regular consistency (see Figure 7).

\subsection{Relative contribution of jaw motion}

The average angle of the first principal component increased significantly with age for both the puree $(\gamma=.86 ; t=2.44 ; d f=10$, $p<.05)$ and regular $(\gamma=.98 ; t=2.68, d f=10, p<.05)$ consistency categories (see Figure 8). A model was also fit with a quadratic trend but accrued similar results in terms of the average change from 9- to 30-months of age across food types and was not significant for either consistency category. The increase in the average angle indicates that the horizontal component of a chewing sequence decreased with age relative to the vertical component.

\subsection{Consistency effects}

3.4.1. Maximum horizontal jaw closing speed

Maximum horizontal speed did not differ across consistencies at the earliest ages studied (9-18 months of age); however, by 24 months of age, a significant difference was detected between the two consistency groups based on the estimates and derived from the HLM analysis and construction of appropriate contrasts. More specifically, maximum horizontal speed was significantly slower for the puree consistency foods than for the regular consistency foods at the ages of 24- $\left[\chi^{2}=9.34, d f=1, p<.01\right]$ and 30months $\left[x^{2}=7.70, d f=1, p<.01\right] .^{2}$

\subsubsection{Maximum vertical jaw closing speed}

The maximum vertical speed did not differ significantly at the earliest ages studied (9 and 12 months of age); however, by 18 months of age a significant difference was detected between the two consistency groups based on the estimates and derived from the HLM analysis and construction of appropriate contrasts. More specifically, at 18 months of age maximum speed was significantly slower for the puree consistency foods than for the regular consistency foods $\left[x^{2}=16.76, d f=1, p<.001\right]$. This significant difference was also detected at $24\left[\chi^{2}=41.20, d f=1, p<.001\right]$ and 30 $\left[\chi^{2}=45.56, d f=1, p<.001\right]$ months of age.

\subsubsection{Relative contribution of jaw motion}

The angle of the first principal component for the puree and regular consistencies did not differ significantly at the earliest stages studied (9-18 months of age); however, by 24 months of age, a significant difference was detected between the two consistency groups based on the estimates and derived from the HLM analysis and construction of appropriate contrasts. Specifically, the angle of the first principal component for regular foods was significantly greater than the angle for puree foods beginning at $24\left[\chi^{2}=4.47\right.$, $d f=1, p<.05]$ and $30\left[\chi^{2}=3.68, d f=1, p=.05\right]$ months of age.

\section{Discussion}

The purpose of this investigation was to quantify age- and consistency-related changes in jaw motion during mastication. The most notable change in jaw movement with age was a general decrease in maximum jaw closing speed observed with the puree consistency. Contrary to our prediction, the relative contribution of the horizontal component of jaw motion decreased significantly with age. These findings do not support a distinct transition from a vertical to a rotary chewing pattern between the ages of 9 and 30 months.

Another key finding was that bolus consistency effects on jaw movements were not evident until 18 months of age. Overall,

2 A nearly significant difference was detected at 18 months of age $\left[\chi^{2}=3.51, d f=1, p=.058\right]$. 


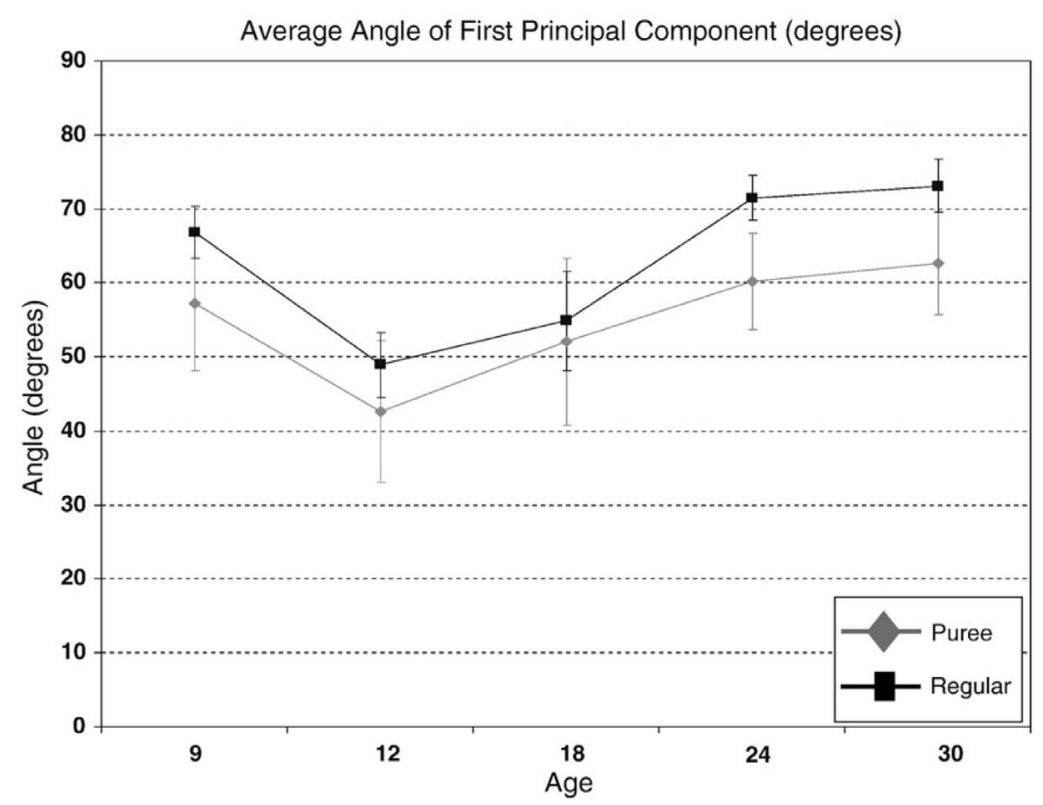

Figure 8. Figure of the average angle of the first principal component from 9-30 months of age across both consistency categories. Note the significant increase in the average angle with an increase in age. Note also that the average angle of the first principal component is generally greater for the regular consistency category than the puree consistency category; however, not statistically so until 24 and 30 months of age. The error bars represent average standard error across participants.

these observations suggest that the development of a rotary chewing pattern, movement speed, and children's sensitivity to bolus consistency have a protracted trajectory during the first two years of life.

\subsection{Age effects}

The age findings suggest that early chewing is characterized by mandibular movement overshoot and that the development of an efficient chewing pattern involves adapting mandibular control to the requirements of different consistencies. The inability to scale movements and force appropriately may be a characteristic feature of immature motor performance. For example, Forssberg and colleagues reported substantial refinement with development in the temporal and force parameters of precision grip [38]. Green and colleagues observed overshoot of mandibular movements during the early phases of speech learning [39]. In developmental studies on reaching, Mathew and Cook [40] and Thelen [41] proposed that arm movement for early reaching is characterized by inaccurate movement and substantial system overshoot. In their studies, arm movement related to reaching became considerably more refined and stable with age.

The horizontal and vertical jaw movements for the puree consistency at 9 months of age were more than twice as fast as those at 30 months of age. The greatest decrease in jaw closing speed for puree consistency occurred between the ages of 9 and 18 months. Although bite force was not studied directly, these findings may indicate that children as young as 18 months of age have learned to regulate bite force to levels needed to breakdown a puree bolus.

The general decrease in jaw closing speed with age may also be due to gradual improvements in bolus management secondary to the emergence of teeth and developmental gains in the control over the lips, cheeks, and tongue. Studies on speech production suggest that young children develop functional control over the jaw earlier than the lips and, possibly, tongue $(39 ; 28)$. Although speculative, children may similarly rely heavily on the jaw during early chewing development. If this is the case, the pattern of jaw motion characteristic of mature chewing might not be expected to emerge until other oral structures (e.g., tongue) effectively assist with bolus preparation.
The general decrease in jaw closing speed with age may be also a response to the emergence of teeth. The compression and sheering forces afforded by teeth decrease the force required to macerate food. Moreover, teeth, particularly the molars, provide a source of biomechanical stability to the jaw, particularly during the occlusal phase of the chewing cycle. Widmer (1992) suggests that, "by the age of 16 months the first primary molars attain occlusal contact" (p. 1252) and the general period for the eruption of deciduous dentition is approximately 20 months [42]. Indeed, across-participant variability in peak jaw speed in the current study was greatest prior to 18 months of age.

\subsection{Consistency effects}

The consistency findings provide converging evidence that the predictive function between masticatory force and bolus consistency is not established prior to 18 months of age. Specifically, differences in horizontal jaw speed for puree and regular consistency were not indicated until 24 months of age; a similar trend was also seen in children as early as 18 months of age for movement in the vertical dimension.

Like all sensorimotor learning, the learning of the relationship between bolus consistency and masticatory force depends on afferent feedback. Bolus consistency characteristics are conveyed via periodontal receptors and mucosa in the oral cavity, which send a signal to the mandibular region of the primary cortex to initiate the appropriate masticatory force [43-45]. Electromyographic differences during maximal occlusal force tasks have been reported in edentulous patients fit with dental prostheses suggesting that changes in afferent feedback can influence masticatory muscle performance [46]. In the current study, the contribution and quality of afferent information may have changed over the course of the study as teeth emerged. Currently, little is known, however, about how the quality of afferent feedback changes as a function of dental eruption in children.

Significant changes in maximum speed with age were not detected for the regular consistency category (in either the horizontal or vertical dimension). This lack of change in movement speed for the regular consistency foods suggest that children are still in the early stages of learning the rotary pattern (necessary for most efficient breakdown of solid foods) prior to 30 months of age. 
4.2.1. The development of a rotary chew pattern was not evident by 30 months

Contrary to our expectations, chewing development was characterized by a marked decrease in horizontal speed. This finding was not consistent with the commonly held assertion that chewing development is characterized by a well-defined transition from a vertical to a rotary chewing pattern (as detailed in the introduction); support for this assertion would have been the observation that horizontal speed of jaw motion during chewing increased with age. Of course, horizontal motion is eventually needed to provide the shearing force to efficiently break down a bolus. One possibility is that the rotary chew pattern does not become evident until after 30 months of age, following the refinement of horizontal mandibular stability or the complete emergence of the molars. This notion is further supported by Takada and colleagues [47], who, citing previous work [48-50] reported that "lateral jaw movement is reflexly modulated by sensory feedback from receptors in or around teeth" (p. 802).

Another possibility is that the discrepancy between the current kinematic-based description of chewing development and prior studies may be because these prior descriptions have relied almost exclusively on video or live visualization of the entire face while children are chewing. According to Green and colleagues, "movement of the mandible may appear deceptively simple" and they suggest that "careful observation reveals characteristic asymmetry in movement paths and wide cycle-to-cycle variations" [2, p. 2704].

Descriptions of jaw motion based on video or live observation analysis procedures may be influenced by motion of other facial structures, which may overshadow the visualization of jaw movements. The impression of jaw motion will also be heavily influenced by the particular view provided by the video camera. For example, sagittal or semi-sagittal orientations, which have been commonly used in the past [24-27, 51, 52], do not provide definitive information about movement along the horizontal dimension. Moreover, because young children move constantly throughout the data collection session, their position relative to the camera can change frequently further complicating observational judgments about jaw movement. In contrast, the 3-dimensional motion capture system used in this investigation provided a means to isolate movement of the jaw from movement of other facial regions (e.g., lips, cheeks, and upper facial regions) regardless of positional changes or camera orientation.

\subsection{Project limitations}

Relative to the other ages, the number of puree chewing trials at 9 months of age was notably smaller (see Table 1). Although the data provide insight into the development of chewing at that young age, the relatively smaller number of data points for this group may not provide a robust representation of the population and may have inflated variability estimates. Future work must be completed to confirm the current findings.

\section{Conclusion}

The findings from this investigation suggest that the development of mandibular control for chewing involves learning to scale movements according to the requirements of different food consistencies. The emergence of a rotary chew pattern was not clearly observed at the ages studied. The current findings provide insight into the typical development of mandibular control for chewing and provide a quantitative foundation from which to better understand childhood feeding and swallowing disorders.

\section{Acknowledgments}

This work has been supported by the National Institutes of Health, National Institute on Deafness and Other Communication Disorders (R03DC004643, R01DC006463, and F31DC006337) and the National Institute of Child Health and Human Development (T32 HD07489). The authors would like to acknowledge Cynthia Didion, Laura Maack, and Kelli Raber for their assistance with data collection and analysis, Dr. Daniel Bolt for statistical consultation, Dr. Gary Weismer for commentary on earlier versions of this manuscript, and the children and their families for their participation.

\section{References}

[1] J. Ahlgren, Mechanism of mastication: a quantitative cinematographic and electromyographic study of mastication movements in children, with special reference to occlusion of the teeth, Acta Odontol Scand 24 (1966), pp. 5-109.

[2] J. R. Green, C. A. Moore, J. L. Ruark, P. R. Rodda, W. T. Morvee and M. J. VanWitzenburg, Development of chewing in children from 12 to 48 months: longitudinal study of EMG patterns, J Neurophysiol 77 (5) (1997), p. 27041.

[3] C. A. Moore and J. L. Ruark, Does speech emerge from earlier appearing oral motor behaviors?, J Speech Hear Res 39 (5) (1996), pp. 1034-1047.

[4] M. Vitti and J. V. Basmajian, Muscles of mastication in small children: an electromyographic study, Am J Orthod 68 (1975), pp. 412-419.

[5] C. A. Moore, A. Smith and R. L. Ringel, Task-specific organization of activity in human jaw muscles, J Speech Hear Res 31 (1988), pp. 670-680.

[6] C. A. Moore, Symmetry of mandibular muscle activity as an index of coordinative strategy, J Speech Hear Res 36 (6) (1993), pp. 1145-1157.

[7] S. E. Morris and M. D. Klein, Pre-feeding skills, A comprehensive resource for feeding development (Second ed), Therapy Skill Builders, United States of America (2000).

[8] J. F. Bosma, Development of feeding, Clin Nutr 5 (5) (1986), pp. 210-218.

[9] J. J. Sheppard and E. D. Mysak, Ontogeny of infantile oral reflexes and emerging chewing, Child Dev 55 (1984), pp. 831-843.

[10] K. A. Pridham, Feeding behavior of 6- to 12-month-old infants: assessment and sources of parental information, J Pediatr 117 (1990), pp. S174-S180.

[11] J. C. Arvedson, Oral-motor and feeding assessment. In: J. C. Arvedson and L. Brodsky, Editors, Pediatric swallowing and feeding: assessment and management, Singular Publishing Group, San Diego (1993), pp. 249-291.

[12] J. C. Arvedson and M. A. Lefton-Greif, Anatomy, physiology, and development of feeding, Semin Speech Lang 17 (4) (1996), pp. 261-268.

[13] J. C. Arvedson, B. Rogers and L. Brodsky, Anatomy, embryology, and physiology. In: J. Arvedson and L. Brodsky, Editors, Pediatric swallowing and feeding: assessment and management, Singular Publishing Group, San Diego (1993), pp. 5-51.

[14] G. L. Pinder and A. S. Faherty, Issues in pediatric feeding and swallowing. In: A. J. Caruso and E. A. Strand, Editors, Clinical management of motor speech disorders in children, Thieme Medical Publishers, Inc, New York (1999), pp. 281-318.

[15] K. Anderson, G. S. Throckmorton, P. H. Buschang and H. Hayasaki, The effect of bolus hardness on masticatory kinematics, J Oral Rehabil 29 (2002), pp. 689-696 System Second ed. Baltimore: Paul H. Brookes Publishing Co. 
[16] S. Filipic and J. Keros, Dynamic influence of food consistency on the masticatory motion, J Oral Rehabil 29 (2002), pp. 492-496.

[17] T. Horio and Y. Kawamura, Effects of texture of food on chewing patterns in the human subject, J Oral Rehabil 16 (1989), pp. 177-183.

[18] Lundeen, H. C. \& Gibbs, C. H., (1982). Advances in occlusion. Great Britain. John Wright \& Sons Ltd. at The Stonebridge Press.

[19] K. Arizumi, Experimental studies concerning the effect of food consistency on masticatory movement in man, J Jpn Prosthodont Soc 33 (1989), pp. 1301-1312.

[20] M. A. Peyron and L. Mioche, Oral assessment of hardness between elastic and plastic products, J Sens Stud 9 (1994), pp. 223-236.

[21] M. A. Peyron, L. Mioche and J. Culioli, Bite force and sample deformation during hardness assessment of viscoelastic models of foods, J Texture Stud 24 (1994), pp. 59-76.

[22] L. Mioche and M. A. Peyron, Bite force displayed during assessment of hardness in various texture contexts, Arch Oral Biol 40 (1995), pp. 415-423.

[23] M. A. Peyron, L. Mioche, P. Renon and S. Abouelkaram, Masticatory jaw movement recordings: a new method to investigate food texture, Food Qual Prefer 7 (1996), pp. 229-237.

[24] E. G. Gisel, Chewing cycles in 2- to 8- year-old normal children: a developmental profile, Am J Occup Ther 42 (1) (1988), pp. 40-46.

[25] E. G. Gisel, Effect of food texture on the development of chewing of children between six months and two years of age, Dev Med Child Neurol 33 (1991), pp. 69-79.

[26] L. M. Schwaab, C. W. Niman and E. G. Gisel, Comparison of chewing cycles in 2-, 3-, 4-, and 5-year-old normal children, Am J Occup Ther 40 (1) (1986), pp. 40-43.

[27] J. L. Schwartz, C. W. Niman and E. G. Gisel, Chewing cycles in 4and 5-year-old normal children: an index of eating efficacy, Am J Occup Ther 38 (1984), pp. 171-175.

[28] J. R. Green, Orofacial movement analysis in infants and young children: new opportunities in speech studies, The Standard 2 (2002), pp. 1-3.

[29] J. Newborg, Battelle developmental inventory (2nd ed. ), Riverside Publishing, Itaska, IL (2005).

[30] Motion Analysis Corporation. (2007). Santa Rosa, CA.

[31] J. R. Green, E. M. Wilson, Y. T. Wang and C. A. Moore, Estimating mandibular motion based on chin surface targets during speech, $J$ Speech Lang Hear Res 50 (4) (2007), pp. 928-939.

[32] National Dysphagia Diet Task Force, National dysphagia diet: standardization for optimal care, American Dietetic Association, Chicago IL (2002).

[33] Green, J. R., \& Nip, I. S. B. (to appear). Organization principals in the development of early speech: catalysts, constraints, and synergy. In B. Maaseen \& P. H. H. M. van Lieshout (Eds. ) Speech motor control: new developments in basic and applied research, NC: Oxford University Press.

[34] U. M. Fietzek, F. Heinen, S. Berweck, S. Maute, A. Hufschmidt and J. Schulte-Mönting et al., Development of the corticospinal system and hand motor function: central conduction times and motor performance tests, Dev Med Child Neurol 42 (4) (2000), pp. 220-227.
[35] J. R. Green and E. M. Wilson, Spontaneous facial motility in infancy: a 3D kinematic analysis, Dev Psychobiol 48 (1) (2006), pp. 16-28.

[36] K. Müller, V. Hömberg and H. G. Lenard, Magnetic stimulation of motor cortex and nerve roots in children. Maturation of corticomotoneuronal projections, Electroencephalogr Clin Neurophysiol 81 (1) (1991), pp. 63-70.

[37] D. J. Ostry, E. Keller and A. Parush, Similarities in the control of the speech articulators and the limbs: kinematics of tongue dorsum movement in speech, J Exp Psychol Hum Percept Perform 9 (4) (1983), pp. 622-636 Aug.

[38] H. Forssberg, A. C. Eliasson, H. Kinoshita, R. S. Johansson and G. Westling, Development of human precision grip I: basic coordination of force, Exp Brain Res 85 (1991), pp. 451-457.

[39] J. R. Green, C. A. Moore, M. Higashikawa and R. W. Steeve, The physiologic development of speech motor control: lip and jaw coordination, J Speech Lang Hear Res 43 (2000), pp. 239-255.

[40] A. Mathew and M. Cook, The control of reaching movements by young infants, Child Dev 61 (4) (1990), pp. 1238-1257.

[41] E. Thelen, Motor development-a new synthesis, Am Psychol 50 (2) (1995), pp. 79-95.

[42] R. P. Widmer, The normal development of teeth, Aust Fam Physician 21 (9) (1992), pp. 1251-1261.

[43] P. G. Dellow and J. P. Lund, Evidence for central timing of rhythmical mastication, J Physiol Lond 215 (1971), pp. 1-13.

[44] J. P. Lund, Mastication and its control by the brain stem, Critical Reviews in Oral Biology \& Medicine 2 (1) (1991), pp. 33-64.

[45] A. J. Thexton, Mastication and swallowing: an overview, Br Dent J 173 (1992), pp. 97-206.

[46] J. L. Gartner, K. Mushimoto, H. P. Weber and I. Nishimura, Effect of osseointegrated implants on the coordination of masticatory muscles: a pilot study, J Prosthet Dent 84 (2) (2000), pp. 185-193.

[47] K. Takada, S. Miyawaki and M. Tatsuta, The effects of food consistency on jaw movement and posterior temporalis and inferior orbicularis oris muscle activities during chewing in children, Arch Oral Biol 39 (9) (1994), pp. 793-805.

[48] P. Schaerer, R. E. Stallard and H. Zander, Occlusal interferences and mastication: an electromyographic study, J Pros Dent 17 (5) (1967), pp. 438-449.

[49] A. G. Hannam, W. W. Wood, R. E. DeCou and J. D. M. Scott, The effects of working-side occlusal interferences on muscle activity and associated jaw movements in man, Archs Oral Biol 26 (1981), pp. 387-392.

[50] K. Takada, Computer-based functional analysis of malocclusions, J Jpn Ortho Soc 51 (1992), pp. 112-123.

[51] E. G. Gisel, L. J. Lange and C. W. Niman, Chewing cycles in 4- and 5-year-old Down's syndrome children: a comparison of eating efficacy with normals, Am J Occup Ther 38 (10) (1984), pp. 666-670.

[52] S. Schwartz, E. G. Gisel, D. Clarke and H. Haberfellner, Association of occlusion with eating efficiency in children with cerebral palsy and moderate eating impairment, J Dent Child 70 (2003), pp. 33-39. 